\title{
Flaming, bright galaxies along the filaments of A 2744
}

\author{
F. Braglia, D. Pierini, and H. Böhringer
}

\begin{abstract}
Max-Planck-Institut für extraterrestrische Physik (MPE), Giessenbachstrasse 1, 85748 Garching, Germany
e-mail: [fbraglia;dpierini;hxb]@mpe.mpg.de
\end{abstract}

Received 27 February 2007 / Accepted 30 April 2007

\section{ABSTRACT}

\begin{abstract}
Context. The existence of a lumpy, large-scale filamentary structure is at the basis of the current paradigm of cosmic formation and evolution of clusters. The star-formation history of galaxies falling into a cluster is altered as a result of the environmental stresses. Aims. We investigate the relation between substructure and properties of the galaxy population in a $30^{\prime} \times 30^{\prime}$ region centred on the massive merging cluster A 2744 at $z \sim 0.3$.

Methods. Multi-object spectroscopy at low resolution and $B V R$ photometry are used to detect the presence of substructure through a Dressler-Schectman analysis and the photometric redshift technique, respectively. Galaxies at the same photometric redshift of the cluster are divided into red and blue according to their distribution in the $B-R$ vs. $R$ colour-magnitude diagram.

Results. We identify two large-scale filaments associated with A 2744. Along these filaments, the blue-to-red galaxy number ratio increases together with the cluster-centric distance but peaks slightly beyond the cluster virial radius. The filaments host a population of bright, large (i.e. more luminous than $R^{\star}$ for the main body of the cluster and with angular sizes of 13-22 $h_{70}^{-1} \mathrm{kpc}$ ) blue galaxies that is hardly found among galaxies lying in a neighbouring low-density environment at the same redshift of the cluster.

Conclusions. These results can be interpreted as a manifestation of galaxy harassment.
\end{abstract}

Key words. galaxies: clusters: general - galaxies: clusters: individual: Abell 2744 - galaxies: clusters: individual: AC 118 cosmology: observations - galaxies: evolution - galaxies: interactions

\section{Introduction}

Detailed numerical simulations show that clusters form at the intersection of filaments and sheets of matter in the evolving large-scale structure of the Universe (e.g., Colberg et al. 1999). These infall-pattern features are correlated in time and appear to be lumpy rather than homogeneous. Thus they define preferred directions from which clusters are fed with lumps of matter.

The existence of extended filamentary structures and voids in the large-scale spatial distribution of local galaxies is known since the advent of redshift surveys (Davis et al. 1982). Later $\mathrm{X}$-ray observations detected the emission from the hot $\left(10^{5}\right.$ $10^{7} \mathrm{~K}$ ) gas in filaments of nearby clusters (Briel \& Henry 1995; Kull \& Böhringer 1999; Scharf et al. 2000; Durret et al. 2003).

In addition, optical observations established the existence of a correlation between the morphology or star-formation rate of a galaxy and the local galaxy density or cluster-centric distance (Dressler 1980; Whitmore et al. 1993; Dressler et al. 1997; Balogh et al. 1999; Poggianti et al. 1999). In particular, the spectral-index analysis for galaxies of the CNOC1 cluster sample at $z \sim 0.3$ demonstrated that the radial increase in starformation activity means that the last episode of star formation occurred more recently in galaxies farthest from the cluster centre (Balogh et al. 1999). Different mechanisms can establish this pattern and, thus, the observed difference in star-formation activity between cluster and field galaxies (e.g., Abraham et al. 1996; Morris et al. 1998; Balogh et al. 1999). They include rampressure stripping by the intracluster medium (ICM) (e.g., Gunn \& Gott 1972; Quilis et al. 2000), close encounters (Barnes 1992), "galaxy harassment" (Moore et al. 1996) and "strangulation" (Larson et al. 1980).
Determining the relative importance of these mechanisms requires probing galaxy properties as a function of the infallregion topology and mass of a cluster. In this pilot study, we investigate A 2744 (AC 118 or RXC J0014.3-3022) out of the REFLEX-DXL (Distant X-ray Luminous) catalogue. This is a homogeneous, unbiased sample drawn from the REFLEX survey (Böhringer et al. 2001) comprising the thirteen most luminous clusters at $z=0.27-0.31$ in the Southern hemisphere, with X-ray $([0.1-2.4] \mathrm{keV})$ luminosities in excess of $10^{45} h_{70}^{-1} \mathrm{erg} \mathrm{s}^{-1}$ (Zhang et al. 2004, 2006). Multiple evidence shows that the main body of A 2744 is a merging system (Boschin et al. 2006 and references therein). In a preliminary analysis (Böhringer at al. 2006), the comparison of X-ray and optical properties suggested the presence of two filamentary structures stemming out of the main body of the cluster. Here we present a more accurate kinematical analysis of the cluster and investigate the galaxy populations associated with these filaments.

\section{Data description and reduction}

Imaging in the $B-, V$-, and $R$-passbands was carried out using the wide-field imager (WFI) on the ESO/MPG-2.2 $\mathrm{m}$ telescope at La Silla, Chile in September 2000, with a seeing of $\sim 1^{\prime \prime}$. The WFI data were reduced using the data reduction system developed for the ESO Imaging Survey (EIS, Renzini \& da Costa 1997) and its associated EIS/MVM image processing library version 1.0.1 (Alambic, Vandame 2004) ${ }^{1}$. Source detection and photometry were performed with SExtractor

\footnotetext{
1 Alambic is a publicly available software designed to automatically transform raw images from single/multi-chip optical/infrared cameras into reduced images for scientific use.
} 
(Bertin \& Arnouts 1996). Source photometry was extracted in fixed circular apertures ( 2 " wide for the determination of photometric redshifts) or in a flexible (Kron-like, Kron 1980) elliptical aperture with a Kron-factor of 2.5. Magnitudes were calibrated to the Johnson-Cousins system using standard stars (Landolt 1992), corrected for galactic extinction (Schlegel et al. 1998), and expressed in the $\mathrm{AB}$ system. The photometric catalogue is complete down to $R \sim 23.5$ and contains objects across a field of $1 / 4$ of a square degree.

Multi-object spectroscopy was performed with VLT-VIMOS in low resolution $(R=200)$ mode (LR-Blue grism) in September 2004. With a slit-width of $1^{\prime \prime}$, the expected uncertainty on the observed velocities is $250-300 \mathrm{~km} \mathrm{~s}^{-1}$. Comparison with previously known redshifts in the same region gives a mean error of $276 \mathrm{~km} \mathrm{~s}^{-1}$, in agreement with with the value estimated by Le Fè et al. 2005 for the same LR-Blue grism. This is enough to establish the membership of a galaxy and the presence of largescale structure for a massive cluster like A 2744. Objects with $I \leq 22.5$ were selected as targets for spectroscopy from VLTVIMOS pre-imaging of a $24^{\prime} \times 22^{\prime}$ field. This limiting magnitude corresponds approximatively to an $I^{\star}+3$ galaxy at the redshift of the cluster (0.3068, see Couch et al. 1998). The adopted selection criterion prevents a bias against cluster members with specific star-formation histories (i.e., colours). Thus, it also prevents the completeness of the spectroscopic sample from being dependent on the local galaxy density, given the known relation between this density and the star-formation rate of a galaxy. The spectroscopic observations provided about 900 spectra. These data were reduced using the dedicated software VIPGI $^{2}$.

Throughout the paper we adopt a $\Lambda$ CDM cosmology where $\Omega_{\mathrm{m}}=0.3, \Omega_{\Lambda}=0.7$, and $h_{70}=H_{0} / 70 \mathrm{~km} \mathrm{~s}^{-1} \mathrm{Mpc}^{-1}=1$.

\section{Analysis}

\subsection{Evidence of large-scale structure}

Spectroscopic redshifts were first converted into velocities according to Danese et al. (1980). We then applied a recursive $3 \sigma$ clipping algorithm to the subsample of galaxies selected in a velocity interval of $10000 \mathrm{~km} \mathrm{~s}^{-1}$ width centred on the known redshift of the cluster. As a result, we identified 134 cluster members. This number was increased by additional 60 cluster members identified from redshifts available in the $N E D$, mostly lying within $1 \mathrm{Mpc}$ from the centre of the cluster. The ensuing mean redshift of the cluster $(0.3068 \pm 0.006)$ is in excellent agreement with previous determinations. The total number of 194 spectroscopic cluster members is twice as large as the number considered in the analysis of Boschin et al. (2006). Furthermore, it probes an area almost four times as large.

We determine a rest-frame velocity dispersion of the cluster equal to $1509 \mathrm{~km} \mathrm{~s}^{-1}$. If A 2744 were a dynamically relaxed system, its velocity dispersion and virial radius ${ }^{3}$ would give a dynamical mass equal to $3.3 \times 10^{15} h_{70}^{-1} M_{\odot}$. However, a Kolmogorov-Smirnov (KS) test reveals that the distribution of radial velocities has only a $0.02 \%$ probability to be Gaussian. This is consistent with A 2744 being a merging system. Combining galaxy velocity and position information (Dressler \& Schectman 1988), we identify two very prominent

${ }^{2}$ VIPGI (VIMOS Interactive Pipeline and Graphical Interface) (Scodeggio et al. 2005) is developed by the VIRMOS Consortium to handle the reduction of the VIMOS data for the VVDS (VIMOS VLT Deep Survey, Le Fèvre et al. 2005).

${ }^{3} R_{\mathrm{Vir}}=2.5 h_{70}^{-1} \mathrm{Mpc}$, from the X-ray analysis of Zhang et al. (2006).

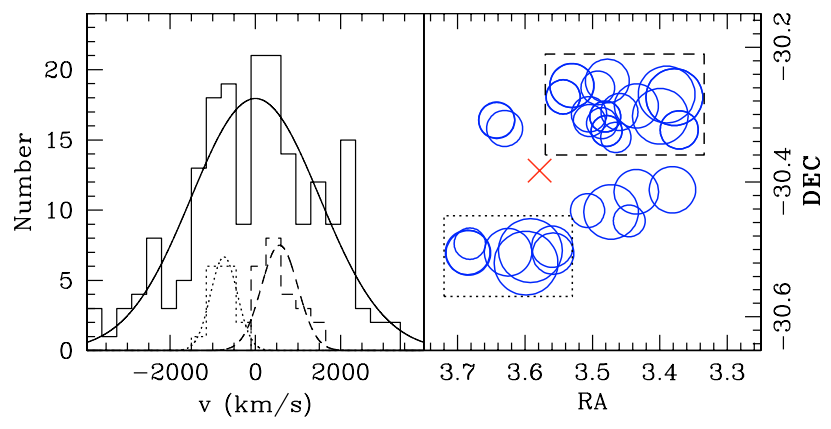

Fig. 1. Left: velocity distribution for different regions of A 2744 , i.e.: the overall system (solid line), with a velocity dispersion of $1509 \mathrm{~km} \mathrm{~s}^{-1}$; the substructure to the NW (dashed line), with a mean velocity of $566 \mathrm{~km} \mathrm{~s}^{-1}$ and a dispersion of $428 \mathrm{~km} \mathrm{~s}^{-1}$; the substructure to the $\mathrm{S}$ (dotted line), with a mean velocity of $-738 \mathrm{~km} \mathrm{~s}^{-1}$ and a dispersion of $314 \mathrm{~km} \mathrm{~s}^{-1}$. Right: Dressler-Schectman test for the spectroscopic cluster members; the dashed- and dotted-line boxes outline respectively the NW and S substructures detected as very significant deviations from the overall velocity distribution. The red cross cross marks the cluster $\mathrm{X}$-ray centre.

substructures, lying at $9.3^{\prime}$ to the NW and $8.2^{\prime}$ to the $\mathrm{S}$ with respect to the cluster main body. These substructures are external but contiguous to those identified by Boschin et al. (2006). They find counterparts in the structures seen in the hot-gas entropy map (Finoguenov et al. 2005) as remarked in Böhringer et al. (2006). In particular (see Fig. 1), we find that the NW structure corresponds to a clump of 23 objects with a velocity distribution characterised by a mean relative velocity of $+566 \mathrm{~km} \mathrm{~s}^{-1}$, a dispersion of $428 \mathrm{~km} \mathrm{~s}^{-1}$ and a positive skew. We picture these galaxies as infalling from the near side of the cluster. Conversely, the $\mathrm{S}$ structure corresponds to a tighter clump of 15 objects, with a mean relative velocity of $-738 \mathrm{~km} \mathrm{~s}^{-1}$ and a dispersion of $318 \mathrm{~km} \mathrm{~s}^{-1}$. This suggests infall from the far side of the cluster.

We run extended Monte Carlo simulations over our spectroscopic sample, reshuffling the redshifts of the objects while maintaining their positions as in Dressler \& Schectman (1988) and adding a random Gaussian error with sigma equal to the dispersion found. We also run the same test by generating a Gaussian velocity distribution. Comparison of the DS-test deviation distributions shows that the inclusion of errors is not sufficient to change the overall behaviour: the two distributions are always consistent, the mean KS statistical parameter being 0.57 , to be confronted with a rejection threshold of 0.88 . Conversely, comparison of the true redshift distribution with the Gaussian case shows that the two distributions differ not only in velocity, but also in the distribution of DS deviations (we obtain a KS parameter of 0.81 , against a threshold for rejection of 0.53 ). The spatial location of the highly deviating peaks does not change position, so they are clearly robust against the size of velocity errors. This is mainly due to the statistical nature of the DS test, which looks for deviations of whole clumps of galaxies by comparing the velocity mean and dispersion of individual groups with the overall velocity dispersion of the cluster. So the effect of errors of individual velocities is smeared down.

In order to map these structures beyond the area covered by spectroscopy, we determine photometric redshifts for the whole photometric catalogue with the HyperZ code (Bolzonella et al. 2000). Photo- $z$ solutions are trained on the available spectroscopic redshifts (about 800 non-stellar objects in the redshift range $[0,0.85])$, so to select the most efficient templates at the cluster redshift. We divide our spectro-photometric sample into red and blue objects on the basis of the cluster red sequence as 


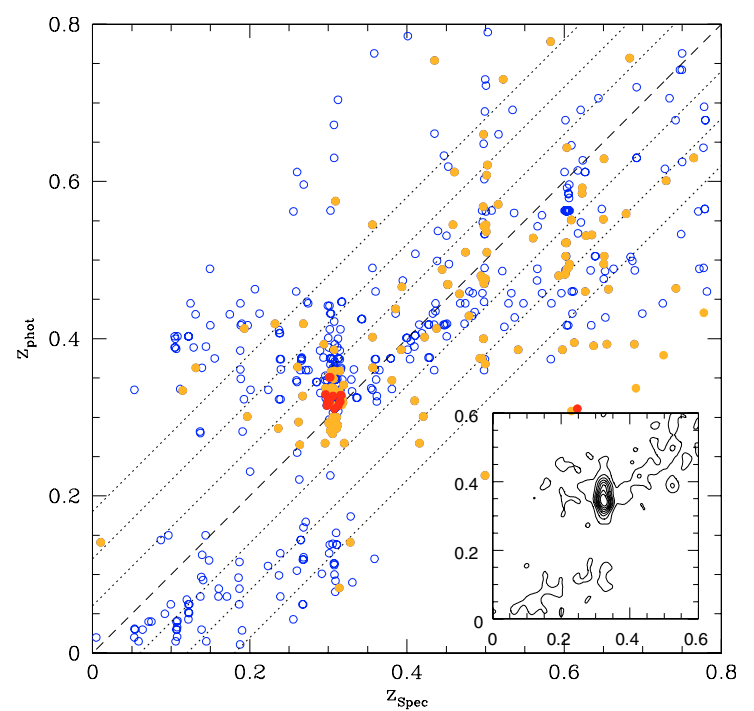

Fig. 2. Comparison of spectroscopic and photometric redshifts. Orange dots: faint red galaxies; red dots: bright red galaxies; blue circles: blue galaxies. The dashed lines show the $1 \sigma, 2 \sigma$ and $3 \sigma$ intervals, where we take as $\sigma$ the spread of the photo- $z$ at the cluster redshift. The lower right insert shows a region of the same distribution as a contour plot to highlight the density (almost 200 points) in the cluster region.

described in Sect. 3.2. Then, we separate objects into bright and faint, where we define as "bright" the galaxies that are equally or more luminous than the characteristic magnitude for the main body of the cluster, i.e., $R^{\star} \sim 19.6 \pm 0.3$ (Busarello et al. 2002). For the resulting 4 catalogues we check independently which templates to use for the photo- $z$ determination, running HyperZ over the same redshift range. Galaxies at the distance of the cluster are then identified as objects in the photo- $z$ range $[0.28,0.40]$. This corresponds to the $1 \sigma$ interval centred on the mean photometric redshift $(0.34)$ of the spectroscopic cluster members. The rms uncertainty is 0.06 at the spectroscopic redshift of A 2744. We see a somewhat wider spread for blue objects, as can be expected; nevertheless, the cluster stands clearly out in the photo- $z$ distribution (see Fig. 2). The $\chi^{2}$ distribution for random objects from the samples exhibits always a single minimum (and thus a single solution).

We check the occurrence of catastrophic failures (i.e., wrong identifications) running HyperZ over the spectro-photometric catalogue with increasing redshift ranges (from [0,0.85] to $[0,6])$. This is done for both red and blue objects independently. No catastrophic failure is found up to $z \sim 1.5$. From this result and the relatively bright magnitude limit of the sample $(R \sim 23.5)$, we adopt a safe range of $[0,1]$ for the photo- $z$ determination across the whole field.

To check the robustness of this assumption against contamination from high- $z$ outliers, we apply the same photometric redshift analysis over a simulated catalogue of objects in the chosen magnitude range and with a suitable redshift distribution. A sample of $\sim 30000$ mock objects is thus created with the HyperZ routine make_catalogue down to $R=23.5$ and with a redshift distribution consistent with deep, magnitude-selected spectroscopic surveys (VVDS-Deep, Le Fèvre et al. 2005) to replicate as much as possible a natural redshift distribution over the field of view.

We run HyperZ with the selected templates over the simulated catalogue constrained to the redshift range $[0,1]$; the result is shown in Fig. 3. While we see (as expected) some contamination from outliers, the wrong identifications mainly lie outside the photometric redshift range of the cluster $(0.28-0.4)$.

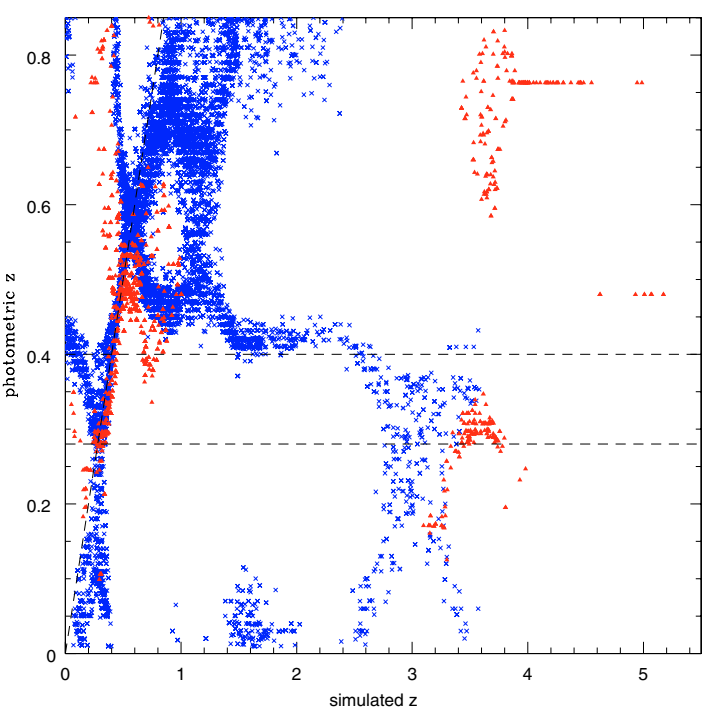

Fig. 3. Simulated contamination from high- $z$ outliers. Red triangles represent old, passively evolving galaxies; blue crosses, star-forming galaxies. The dashed lines mark the cluster region in redshift space. Three main sources of contamination are identified: high- $z$ red galaxies (105 objects, contamination of $6.9 \%$ ) ; high- $z$ blue galaxies (185 objects, contamination of 1.7\%); and foreground blue galaxies (349 objects, contamination of $3.2 \%$ ).

In this simulation we did not take into account the presence of the cluster at $z=0.3068$, as we want to assess the pure contamination from background objects. Comparing the number of interlopers with the photometric cluster members $(5759$ objects within the cluster photo- $z$ range) shows that the contamination is still quite low (1.8\% for high- $z$ red objects, $3.2 \%$ for high- $z$ blue objects and $6.1 \%$ for blue foreground objects). Thus, we conclude that the effect of contamination in the cluster redshift range is negligible.

For the 5759 objects at the distance of the cluster, divided into a red subsample and a blue one (see Sect. 3.2), we determine separate number density maps. As Fig. 4 shows, the bulk of the red objects sits in the main body of the cluster, as expected. However, two extensions of red objects are clearly seen towards the same NW and S directions defined by the structures identified by the Dressler-Schectman analysis. On the other hand, the blue objects are almost evenly spread across the imaged region. A closer look reveals a chain of highly significant overdensities of blue objects stemming out of the S extension of red objects and turning to the SW. This is very suggestive of an extended, lumpy filament as simulations predict. An extended filament in the NW direction is less evident.

\subsection{Star formation along the large-scale structure}

As a zero-point for the description of the star-formation activity in the region of A 2744 , we take the red sequence of the likely massive, old, passively evolving galaxies in the $B-R$ vs. $R$ colour-magnitude diagram. Here it is defined as the locus of galaxies with $17 \leq R \leq 20$ and $B-R \sim 2.2$ within a clustercentric distance of $3^{\prime}$ (i.e., about $800 \mathrm{kpc}$ ). Then we divide the total sample of 5759 galaxies at the distance of the cluster into a red subsample and a blue one. An object is classified as blue if its $B-R$ colour is bluer than that of a mean red-sequence object with the same $R$-magnitude at more than the $3 \sigma$ level.

Furthermore, we define two circular sectors with a $60^{\circ}$ aperture and radii from $3^{\prime}$ to $15^{\prime}$ which encompass the two filaments. 


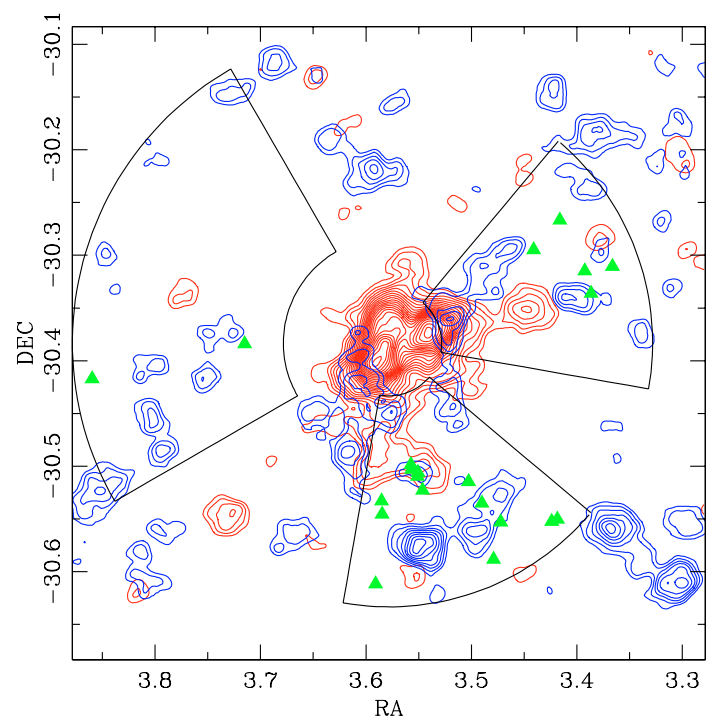

Fig. 4. Density contours for red and blue galaxies at the distance (photo- $z$ ) of the cluster, in multiples of background rms. The red objects density map shows the non-relaxed main body of the cluster and the head of the filamentary structures towards the S and NW. The blue objects map shows the presence of regions of high density in the outskirts of the cluster. In particular, two high density regions toward the $\mathrm{S}$ and NW lie on the extension of the substructures emerging from the cluster main body. The regions delimiting the two filaments and the "field" are also shown. Triangles mark the position of the luminous blue galaxies.

A third circular sector with a $90^{\circ}$ aperture and radii from $6^{\prime}$ to $18^{\prime}$ defines an area equivalent to the sum of the previous two regions where no structure is evident. This third region represents the comparison "field" at the same redshift of the cluster. Down to the completeness limit, there are 570 and 500 objects in the $\mathrm{S}$ and NW circular sectors, respectively, against a total of 796 in the field. Hence the significance of the average overdensity is equal to $9 \sigma$ and $5 \sigma$ for the S and NW circular sectors, respectively, and to $10 \sigma$ overall. The overdensity is driven by faint galaxies: $15 \sigma$ and $11 \sigma$ for the red and blue galaxies with $R^{\star}<R \leq 23.5$, respectively, against $6 \sigma$ and $5 \sigma$ for the red and blue galaxies with $R \leq R^{\star}$, respectively.

Figure 5 reproduces the $B-R$ vs. $R$ colour-magnitude diagram for galaxies in these three regions. Luminous, red galaxies exhibit similar, tight colour-magnitude relations. In the higherdensity regions, red galaxies with $17 \leq R \leq 20$ define a redsequence that is shifted bluewards by $0.012 \pm 0.003$ mag with respect to the red-sequence in the main body of the cluster. As for the population of blue galaxies, a KS-test run for bins of $0.5 R$-mag and the full $B-R$ colour range shows a very significant $(9 \sigma)$ overabundance of galaxies with $17.6 \leq R \leq 19.3$ and $0.8 \leq B-R \leq 1.3$ in the higher-density regions. This does not simply mirror the overabundance of luminous, blue galaxies there.

These bright, blue (and, thus, star-forming) galaxies are large systems, as confirmed visually by eye-ball estimation of their angular sizes to about $3-5^{\prime \prime}$ (i.e. about $13-22 h_{70}^{-1} \mathrm{kpc}$ ). They lie mostly in or close to overdensities of blue objects and around or beyond the cluster virial radius $R_{\text {Vir }}$ (about $9^{\prime}$ or $2.5 \mathrm{Mpc}$ ). Out of these 19 luminous, blue galaxies, only three can be part of interacting pairs, whereas all are surrounded by several small companions.

Finally, we note that the relative fraction of blue galaxies increases moving outwards along the regions containing the filaments (see Fig. 4), possibly because the number of blue galaxies

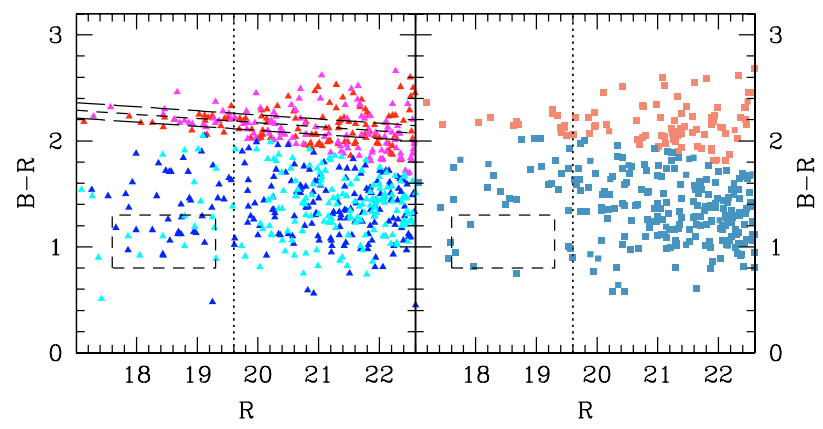

Fig. 5. Colour-magnitude diagram for the galaxies in the filaments (left) or in the field (right). The population of the two filaments is represented in different colours: red objects appear in red (S filament) and magenta (NW filament), blue objects in blue (S filament) and cyan (NW filament). The signature of a red sequence can clearly be seen for the filaments, as well as the peculiar population of blue galaxies, in the dashed box; this population is not matched in the field. In each panel, the dashed vertical line marks the value of $R^{\star}$ for the main body of the cluster.

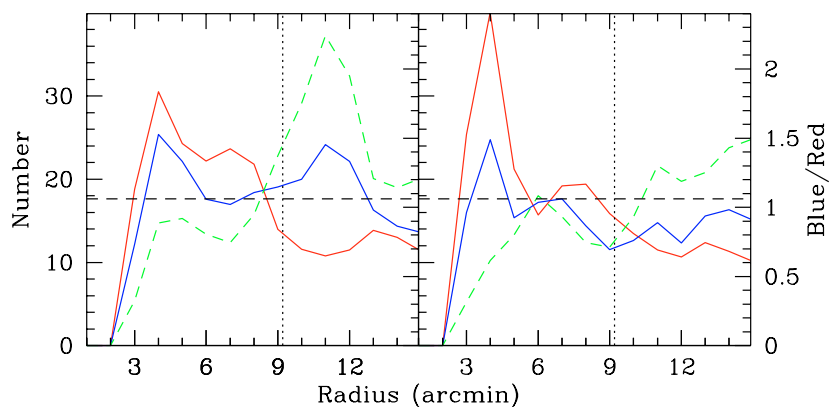

Fig. 6. Radial trend of the galaxy population in the filaments, in bins of $1^{\prime}$ width (left: S filament; right: NW filament. The galaxy number reads on the left margin, the blue-to-red ration on the right one). Along with the trend of the two populations (red and blue lines), the ratio of blue-to-red galaxies is shown (dashed green line) and compared with the mean value in the field (horizontal dashed line). Beyond the virial radius (vertical dotted line), a sudden increase in the ratio is seen, up to twice the field value.

decreases less rapidly than the number of red galaxies. However, in the southern region a sudden increase of blue galaxies contributes to the peak in the blue-to-red galaxy number ratio at a cluster-centric distance of about $1.2 R_{\mathrm{Vir}}$. This peak is significant with respect to the relative fraction of blue galaxies in the field.

\section{Discussion and conclusions}

In conjunction with the seminal study of Böhringer et al. (2006), our analysis strongly suggests the presence of two large-scale filaments associated with the massive merging cluster A 2744 at $z=0.3068$. Similar evidence exists for other clusters at intermediate redshifts (Kodama et al. 2001; Ebeling et al. 2004). On the other hand, the existence of a lumpy, large-scale filamentary structure is at the basis of the current paradigm of cosmic formation and evolution of clusters (e.g., Colberg et al. 1999).

We investigate star formation in galaxies selected in two regions encompassing the large-scale filaments of A 2744 and in an equivalent, wide region where the density of galaxies at the (photometric) redshift of the cluster is significantly lower (the field). The overdensity in the filaments is driven by the population of galaxies fainter than $R^{\star}$ (as determined by Busarello et al. (2002) in the main body of the cluster), whatever their $B-R$ colour. However, the blue-to-red galaxy number ratio 
increases with increasing cluster-centric distance along the filaments but peaks at about $1.2 R_{\mathrm{Vir}}$. This result is consistent with and complementary to the established existence of a radial dependence of azimuthal averages of cluster galaxy properties like colours or emission-line strengths (e.g. Balogh et al. 1999; Lewis et al. 2002; Gómez et al. 2003; Finn et al. 2005). It is also consistent with the result that the relative fraction of blue galaxies peaks between 1 and $2 R_{V i r}$ in the nearby Shapley and Pisces-Cetus superclusters (Haines et al. 2006; Porter \& Raychaudhury 2006).

A 2744 is a merging cluster, where the mass-ratio of the subcomponents is about 3:1 (Boschin et al. 2006). A qualitative comparison between the Chandra X-ray map (Kempner \& David 2004), exhibiting a signature of a bow-shock, and recent model renditions of shock heating in cluster mergers (McCarthy et al. 2007) suggests that merging started less than 2 Gyr before the observed epoch. The primary shock could not travel to distances as large as the virial radius of the cluster. Hence the galaxy population along the filaments of A 2744 has not been affected by this large-scale merging event.

In their seminal spectroscopic study of the galaxy population in the core region of this cluster, Couch et al. (1998) find that the majority of star-forming galaxies is generally made of systems involved in major mergers but of modest luminosity, even in this brightened phase. These galaxies are interpreted as the progenitors of dwarf systems once they fade. Interestingly, we find that the filaments of A 2744 host a population of luminous, large (i.e. brighter than $R^{\star}$ and with sizes of $13-22 h_{70}^{-1} \mathrm{kpc}$ ), blue galaxies that hardly exists in the field. Hence these galaxies can either retain or gain gas while they are falling into the main body of the cluster.

Galaxy harassment (Moore et al. 1996) is a suitable physical explanation for the enhanced star formation inferred for these sparse, luminous, blue galaxies found at distances close to the virial radius of A 2744, where the density of the ICM is low. As envisioned by Moore et al. (1999), high-surface brightness disc galaxies and galaxies with luminous bulges do not experience a significant removal of material nor a transformation in Hubble type under the influence of high-speed, close encounters with substructure (including bright galaxies) and strong tidal shocks from the global cluster potential. However, their discs will be heated and undergo instabilities that can funnel gas (if any initially) into the central regions (Lake et al. 1998). Galaxy harassment can generate morphological instabilities in galaxies already at the outskirt of a cluster (Moore et al. 1998; Mastropietro et al. 2005). In the core of the cluster, ram pressure can strip all the residual gas from discs and drive a morphological transformation into S0's, as speculated by Moore et al. (1999). Alternatively, this can happen there once the shock associated with the large-scale merging decelerates and discs can cross it (see Roettiger et al. 1996). Therefore, the luminous, blue galaxies along the filaments of A 2744 can be analogous to the progenitors of the $\mathrm{S} 0-\mathrm{Sb}$ galaxies in the core of the cluster that completed their last major episode of star formation 1-2 Gyr before the observed epoch (Couch et al. 1998).
Acknowledgements. This research has made use of the NASA/IPAC Extragalactic Database (NED) which is operated by the Jet Propulsion Laboratory, California Institute of Technology, under contract with the National Aeronautics and Space Administration. D.P. acknowledges a stimulating discussion with G. Lake. F.B. wants to thank A. Biviano for useful discussions and suggestions.

\section{References}

Abraham, R. G., Smecker-Hane, T. A., Hutchings, J. B., et al. 1996, ApJ, 471, 694

Balogh, M. L., Morris, Simon, L., Yee, H. K. C., et al. 1999, ApJ, 527, 54

Barnes, J. E. 1992, ApJ, 393, 484

Bertin, E., \& Arnouts, S. 1996, A\&AS, 117, 393

Böhringer, H., Schuecker, P., Guzzo, L., et al. 2001, A\&A, 369, 826

Böhringer, H., Braglia, F., Pierini, D., et al. 2006, The Messenger, 123, 49

Bolzonella, M., Miralles, J.-M., \& Pelló, R. 2000, A\&A, 363, 476

Boschin, W., Girardi, M., Spolaor, M., \& Barrena, R. 2006, A\&A, 449, 461

Briel, U. G., \& Henry, J. P. 1995, A\&A, 302, L9

Busarello, G., Merluzzi, P., La Barbera, F., Massarotti, M., Capaccioli, M., et al. 2002, A\&A, 389, 787

Colberg, J. M., White, S. D. M., Jenkins, A., \& Pearce, F. R. 1999, MNRAS, 308,593

Couch, W. J., Barger, A. J., Smail, I., Ellis, R. S., Sharples, R. M., et al. 1998, ApJ, 497, 188

Danese, L., de Zotti, G., \& di Tullio, G. 1980, A\&A, 82, 322

Davis, M., Huchra, J., Latham, D. W., \& Tonry, J. 1982, ApJ, 253, 423

Dressler, A. 1980, ApJ, 236, 351

Dressler, A., \& Shectman, S. A. 1988, AJ, 95, 985

Dressler, A., Oemler, A. Jr., Couch, W. J., et al. 1997, ApJ, 490, 577

Durret, F., Lima Neto, G. B., Forman, W., \& Churazov, E. 2003, A\&A, 403, L29

Ebeling, H., Barrett, E., \& Donovan, D. 2004, ApJ, 609, L49

Finn, R. A., Zaritsky, D., McCarthy, D. W. Jr., et al. 2005, ApJ, 630, 206

Finoguenov, A., Böhringer, H., \& Zhang, Y.-Y. 2005, A\&A, 442, 827

Gómez, P. L., Nichol, R. C., Miller, C. J., et al. 2003, ApJ, 584, 210

Gunn, J. E., \& Gott, J. R., III 1972, ApJ, 176, 1

Haines, C. P., Merluzzi, P., Mercurio, A., et al., 2006, MNRAS, 371, 55

Kempner, J. C., \& David, L. P. 2004, MNRAS, 349, 385

Kodama, T., Smail, I., Nakata, F., Okamura, S., Bower, R. G., et al. 2001, ApJ, 562 , L9

Kron, R. G. 1980, ApJS, 43, 305

Kull, A., \& Böhringer, H. 1999, A\&A, 341, 23

Lake, G., Katz, N., \& Moore, B. 1998, ApJ, 495, 152

Landolt, A. U. 1992, AJ, 104, 340

Larson, R. B., Tinsley, B. M., \& Caldwell, C. N. 1980, ApJ, 237, 692

Le Fèvre, O., Vettolani, G., Garilli, B., et al. 2005, A\&A, 439, 845

Lewis, I., Balogh, M., De Propris, R., et al. 2002, MNRAS, 334, 673

Mastropietro, C., Moore, B., Mayer, L., et al. 2005, MNRAS, 364, 607

McCarthy, I. G., et al. 2007 [arXiv: astro-ph/0701335]

Moore, B., Katz, N., Lake, G., Dressler, A., Oemler, A., et al. 1996, Nature, 379, 613

Moore, B., Lake, G., \& Katz, N. 1998, ApJ, 495, 139

Moore, B., Lake, G., Quinn, T., \& Stadel, J. 1999, MNRAS, 304, 465

Morris, S. L., Hutchings, J. B., Carlberg, R. G., et al. 1998, ApJ, 507, 84

Poggianti, B., Smail, I., Dressler, A., et al. 1999, ApJ, 518, 576

Porter, S. C., \& Raychaudhury, S. 2006, MNRAS, 375, 1409

Quilis, V., Moore, B., \& Bower, R. 2000, Science, 288, 1617

Renzini, A., \& da Costa, L. 1997, The Messenger, 87, 23

Roettiger, K., Burns, J. O., \& Loken, C. 1996, ApJ, 473, 651

Scharf, C., Donahue, M., Voit, G. M., Rosati, P., Postman, M., et al. 2000, ApJ, 528, L73

Schlegel, D. J., Finkbeiner, D. P., \& Davis, M. 1998, ApJ, 500, 525

Scodeggio, M., Franzetti, P., Garilli, B., et al. 2005, PASP, 117, 1284

Vandame, B. 2004, Ph.D. Thesis, University of Nice-Sophia Antinopolis

Whitmore, B. C., Gilmore, D. M., \& Jones, C. 1993, ApJ, 407, 489

Zhang, Y.-Y., Finoguenov, A., Böhringer, H., et al. 2004, A\&A, 413, 49

Zhang, Y.-Y., Böhringer, H., Finoguenov, A., et al. 2006, A\&A, 456, 55 